\title{
Opção terapêutica para ansiedade: o uso da Cannabis sativa é uma alternativa
}

\section{farmacológica?}

Therapeutic option for anxiety: is the use of Cannabis sativa a pharmacological alternative?

Opción terapéutica para la ansiedad: el uso de Cannabis sativa es una alternativa farmacológica?

Recebido: 07/11/2021 | Revisado: 16/11/2021 | Aceito: 20/11/2021 | Publicado: 01/12/2021

\author{
Adriana Rosa Bueno \\ ORCID: https://orcid.org/0000-0002-6906-1149 \\ Universidade Nilton Lins, Brasil \\ E-mail:17010737@uniniltonlins.edu.br \\ Jessica Vanina Ortiz \\ ORCID: https://orcid.org/0000-0001-7214-6816 \\ Universidade Nilton Lins, Brasil \\ E-mail: ortiz.jvm@gmail.com
}

\begin{abstract}
Resumo
A OMS (Organização Mundial de Saúde) classifica a ansiedade como: transtorno de ansiedade generalizada, transtorno do pânico, fobias sociais ou específicas e transtorno de ansiedade social (SAD). Todos os tipos compartilham sintomas comuns, incluindo sentimentos de mal-estar, pânico e medo, problemas de sono, não ser capaz de ficar calmo, estar com frio e / ou suando, falta de ar, palpitações cardíacas, boca seca, náusea, e prevenção de situações, estes transtornos acabam prejudicando a qualidade de vida de muitas pessoas e levando muita das vezes a cometer suicídios. $\mathrm{O}$ objetivo do presente estudo foi descrever a eficácia do uso do canabidiol para tratamento da ansiedade, descrever os mecanismos fisiológicos e seus efeitos adversos. Para isso foi realizado uma pesquisa bibliográfica através de artigos publicados e atuais onde foi possível analisar o seus efeitos em animais e humanos, os estudos foram realizados em uma população com idades entre 18-37 anos com transtorno de ansiedade social, fobia de falar em público e pessoas com transtorno pós-traumático causado por algum trauma na infância. Os resultados mostraram que o canabidiol pode ser uma potente opção para o tratamento da ansiedade, sua eficácia em relação à dosagem, vias de administração e efeitos adversos mostraram resultados significantes. O presente estudo demonstra que mais estudos clínico e controlado por placebo são necessários para demonstrar a eficácia da canabis.
\end{abstract}

Palavras-chave: Canabis; Canabinóides; Agendamento de drogas; Reposição de medicação; Ansiedade.

\begin{abstract}
The WHO (World Health Organization) classifies anxiety as: generalized anxiety disorder, panic disorder, social or specific phobias and social anxiety disorder (SAD). All types share common symptoms, including feelings of malaise, panic and fear, sleep problems, not being able to stay calm, being cold and/or sweating, shortness of breath, heart palpitations, dry mouth, nausea, and prevention of situations, these disorders end up harming the quality of life of many people and often leading to suicide. The aim of the present study was to describe the effectiveness of using cannabidiol for the treatment of anxiety, to describe the physiological mechanisms and its adverse effects. For this, a literature search was carried out through published and current articles where it was possible to analyze its effects on animals and humans, the studies were carried out in a population aged 18-37 years with social anxiety disorder, public speaking phobia and people with post-traumatic disorder caused by childhood trauma. The results showed that cannabidiol can be a powerful option for the treatment of anxiety, its effectiveness in relation to dosage, administration routes and adverse effects showed significant results. The present study demonstrates that more clinical and placebo-controlled studies are needed to demonstrate the effectiveness of cannabis.
\end{abstract}

Keywords: Cannabis; Cannabinoids; Drug scheduling; Replacement of medication; Anxiety.

\section{Resumen}

La OMS (Organización Mundial de la Salud) clasifica la ansiedad en: trastorno de ansiedad generalizada, trastorno de pánico, fobias sociales o específicas y trastorno de ansiedad social (SAD). Todos los tipos comparten síntomas comunes, que incluyen sensación de malestar, pánico y miedo, problemas para dormir, no poder mantener la calma, tener frío y / o sudoración, dificultad para respirar, palpitaciones cardíacas, sequedad de boca, náuseas y prevención de situaciones, estos Los trastornos terminan perjudicando la calidad de vida de muchas personas y, a menudo, conducen al suicidio. El objetivo del presente estudio fue describir la efectividad del uso de cannabidiol para el tratamiento de la ansiedad, describir los mecanismos fisiológicos y sus efectos adversos. Para ello, se realizó una búsqueda bibliográfica a través de artículos publicados y actuales donde fue posible analizar sus efectos en animales y humanos, los estudios se realizaron en una población de 18-37 años con trastorno de ansiedad social, fobia a hablar en público. Y personas con trastorno postraumático causado por un trauma infantil. Los resultados mostraron que el cannabidiol puede ser una 
opción poderosa para el tratamiento de la ansiedad, su efectividad en relación a la dosis, vías de administración y efectos adversos arrojó resultados significativos. El presente estudio demuestra que se necesitan más estudios clínicos y controlados con placebo para demostrar la eficacia del cannabis.

Palabras clave: Cannabis; Cannabinoides; Programación de medicamentos; Reemplazo de medicación; Ansiedad.

\section{Introdução}

O transtorno de ansiedade é um dos problemas mentais mais prevalentes no mundo, levando a altos custos sociais e económico. A ansiedade é caracterizada por antecipação excessiva de ameaças futuras e acompanhada por medo e pensamentos imoderados, uma resposta emocional de amedrontamento recente. O medo e a ansiedade constante levam a distúrbios comportamentais associados a ataques de pânico, comportamento de isolação e diminuição da sensação de bem-estar, levando para relacionamentos conturbados, conflito familiar, qualidade de vida baixa, aumento das taxas de desemprego e elevado risco de suicídio (Laboissiére,2017; Wright et al., 2020)

Os sintomas relacionados à ansiedade incluem: taquicardia, tensão muscular, dificuldades respiratórias, dificuldade em falar em público, dores de estômago e sudorese. A ansiedade excessiva pode levar a problemas como a depressão, uso abusivo de álcool e uso abusivo de substâncias, incluindo medicamentos psicotrópicos. O desenvolvimento de transtornos de ansiedade também está ligado a certos fatores de risco, incluindo sexo feminino, baixo nível socioeconômico, riscos ambientais e maus tratos durante a infância (Alves et al., 2021)

Atualmente os tratamentos farmacológicos disponíveis incluem inibidores de recaptação de serotonina, inibidores de recaptação de serotonina e noradrenalina, benzodiazepínicos, inibidores da monoamina oxidase, antidepressivos tricíclicos, anticonvulsivantes e antipsicóticos são os mais usados para tratar a ansiedade e transtorno de pânico (TP). Esses medicamentos possuem taxas de respostas limitadas e os efeitos adversos também podem limitar a tolerabilidade e adesão ao tratamento, efeitos colaterais como: disfunção erétil, incapacidade, sonolência e abstinência podem levar a limitações dos tratamentos atuais, o que colocam uma prioridade no desenvolvimento de novos tratamentos (Blessing et al., 2015)

O uso terapêutico de alguns compostos da planta Cannabis sativa, conhecida como maconha tem atraído grande interesse, especialmente para o tratamento de transtornos neuropsiquiátricos devido à relativa falta de eficácia dos tratamentos atuais. Os principais fitocanabinóides como, tetra-hidrocanabidiol (THC) e canabidiol (CBD) são os principais compostos utilizado da planta. O CBD apresenta um interessante perfil farmacológico sem potencial para se tornar uma droga de abuso, ao contrário do THC que é o responsável pelos efeitos eufóricos (Blessing et al., 2015)

A C. sativa tem aproximadamente 120 canabinóides identificados e classificados com base em sua estrutura química. O tetra-hidrocanabidiol (THC), cannabigerol, cannabicromeno, canabidiol (CBD), canabinodiol, canabielsoína, canabinol, canabitriol são alguns dos principais grupos de compostos presente na planta, o THC foi caracterizado por Gaoni e Mechoulam em 1964, substância responsável pelas propriedades de reforço da canabis. O CBD foi isolado pela primeira vez por Adams e Cols em 1940 (Gutiérres et al., 2020)

Este estudo buscou responder o seguinte problema de pesquisa: qual a importância do efeito farmacológico do canabidiol?. Estudo feito para avaliar o efeito do CBD em adolescentes com idade de 18-19 anos com TAS (Transtorno de ansiedade social) e transtorno de personalidade esquiva receberam em um estudo duplo-cego o óleo de canabis, uma parte recebeu uma dose de CBD e a outra recebeu placebo. Foram medidos os sintomas de TAS no início e no final do período de tratamento usando um questionário de avaliação do medo e da Escala Ansiedade Social de Liebowitz. O CBD diminuiu significativamente a ansiedade medida por ambas as escalas. Os resultados indicaram que o canabidiol pode ser uma opção para tratar a ansiedade social. (Masataka, 2019) 
Estudos avaliaram o efeito farmacológico e fisiológico do canabidiol, durante o estudo não houve alterações como: pressão arterial, frequência cardíaca, temperatura e distúrbios bioquímicos. Também não foram detectados problemas gastrointestinais como êmese ou disenteria (Iffland \& Grotenhermen., 2017)

Esta revisão bibliográfica teve como objetivo: Descrever a eficácia do uso do canabidiol para o tratamento da ansiedade, descrever os mecanismos fisiológicos do canabidiol e apontar os efeitos colaterais mais comuns do uso.

\section{Metodologia}

A busca será realizada a partir de revisão bibliográfica com base nos artigos selecionados com maior relevância para o estudo. Serão selecionados artigos pesquisados de agosto a novembro completo e publicados entre 2015 e 2021 . A partir dos critérios estabelecidos. Critérios de inclusão: artigos escritos em inglês e/ou português, que tenham como tipo de estudo: ensaios clínicos randomizados, coorte e/ou caso-controle com disponibilidade de texto na íntegra. Critérios de exclusão: livros, teses e dissertações, além de artigos com mais de cinco anos de publicação. A busca bibliográfica será feita através dos bancos de dados: Google Acadêmico, pubMed e Scielo. Para isso serão utilizados os descritores conforme demonstrados no Quadro 1.

Quadro 1 - Descritores de busca de acordo com o banco de dados

\begin{tabular}{|l|l|l|}
\hline \multicolumn{1}{|c|}{ Google Acadêmico } & \multicolumn{1}{|c|}{ Pub Med } & \multicolumn{1}{c|}{ SciElo } \\
\hline Cannabis Sativa, e o uso do canabidiol. & Reações adversas do canabidiol. & Uso da canabis, na ansiedade. \\
\hline Uso terapêutico do canabidiol. & $\begin{array}{l}\text { Canabidiol VS tetra- } \\
\text { hidrocanabidiol. }\end{array}$ & Tratamentos disponíveis para ansiedade. \\
\hline
\end{tabular}

Fonte: Autores.

\section{Resultados e Discussão}

Ao analisar as publicações sobre o tema e as repercussões sobre o uso da canabis em pacientes com sintomas de ansiedade, buscou-se identificar a percepção dos autores em relação a sua eficácia e como opção terapêutica, no quadro abaixo foi selecionado alguns autores, tipos de estudo e resultado alcançado por eles.

Quadro 2 - Tipos de estudos e resultado realizados pelos autores.

\begin{tabular}{|c|c|c|}
\hline Autor & Estudo & Resultado \\
\hline $\begin{array}{l}\text { Blessing et al., } \\
2015 \text {. }\end{array}$ & $\begin{array}{l}\text { Estudo clínico experimental em } \\
\text { humano, clínico e epidemiológico. }\end{array}$ & $\begin{array}{l}\text { Evidências pré-clínicas apoiam fortemente o CBD como um } \\
\text { tratamento para transtorno de ansiedade. }\end{array}$ \\
\hline $\begin{array}{l}\text { Shannon et al., } \\
2016 .\end{array}$ & $\begin{array}{l}\text { Estudo de caso com paciente } \\
\text { diagnosticada com ansiedade causada } \\
\text { por um trauma na infância e o tratamento } \\
\text { com óleo de canabidiol. }\end{array}$ & $\begin{array}{l}\text { O uso de canabidiol } 25 \mathrm{mg} \text { antes de dormir e } 6 \text { a } 12 \mathrm{mg} \text { spray } \\
\text { sublingual durante o dia em caso de crises demonstrou um } \\
\text { aumento gradual na qualidade de sono e diminuição da } \\
\text { ansiedade em cinco meses. }\end{array}$ \\
\hline $\begin{array}{l}\text { Iffland \& } \\
\text { Grotenhermen, } \\
2017 .\end{array}$ & $\begin{array}{l}\text { Estudos clínicos realizados em humanos } \\
\text { para avaliar a segurança, efeitos } \\
\text { colaterais e potenciais interações com } \\
\text { outros medicamentos. }\end{array}$ & $\begin{array}{l}\text { O perfil de segurança foi favorável em relação ao uso do CBD } \\
\text { em humanos e foi confirmado pela pesquisa revisada. }\end{array}$ \\
\hline $\begin{array}{l}\text { MacCallum \& } \\
\text { Russo, } 2018 .\end{array}$ & $\begin{array}{l}\text { Desafio na hora de prescrever, para } \\
\text { alguns médicos e a polemica em relação } \\
\text { ao mau uso e a falta de informação sobre } \\
\text { o medicamento. }\end{array}$ & $\begin{array}{l}\text { Dados concisos sobre Farmacologia do canabis relacionada ao } \\
\text { tetra-hidrocanabinol (THC), canabidiol (CBD), métodos de } \\
\text { administração (tabagismo, vaporização, oral) e } \\
\text { recomendações de dosagem. }\end{array}$ \\
\hline
\end{tabular}




\begin{tabular}{|c|c|c|}
\hline Diest et al., 2019. & $\begin{array}{l}\text { Este estudo examinou o uso de cannabis } \\
\text { no afeto positivo e afeto negativo em } \\
\text { humanos se diminui em situações sociais } \\
\text { ou aumenta sobre o uso de canabis. }\end{array}$ & $\begin{array}{l}\text { A ansiedade social foi significativamente reduzida, o resultado } \\
\text { foi positivo. }\end{array}$ \\
\hline $\begin{array}{l}\text { Gutiérres et al., } \\
2020 .\end{array}$ & $\begin{array}{l}\text { Estudo clinico aborda os efeitos } \\
\text { ansiolíticos, antidepressivos e anti- } \\
\text { psicóticos do CBD com animais } \\
\text { camundongo e humanos. }\end{array}$ & $\begin{array}{l}\text { O CBD alivia a ansiedade induzida pelo THC em voluntários } \\
\text { saudáveis do sexo masculino. Posteriormente, estudos } \\
\text { adicionais duplo-cegos avaliaram ainda mais os efeitos do } \\
\text { CBD em voluntários saudáveis. }\end{array}$ \\
\hline Sakal et al., 2021. & $\begin{array}{l}\text { Uso da cannabis para tratamento de } \\
\text { doenças primárias mais comumente } \\
\text { relatadas: dor crônicas }(55,6 \%), \\
\text { transtornos de ansiedade }(32,0 \%) \text { e } \\
\text { insônia e depressão. }\end{array}$ & $\begin{array}{l}678 \text { pacientes fizeram parte do estudo, } 32,0 \% \text { relatou sofrer de } \\
\text { insônia e ansiedade e } 55,6 \% \text { relatou ter dor crônica. }\end{array}$ \\
\hline $\begin{array}{l}\text { McFadden \& } \\
\text { Malone, } 2020 .\end{array}$ & $\begin{array}{l}\text { Uma percepção sobre o uso médico e } \\
\text { abuso potencial de CBD e THC. }\end{array}$ & $\begin{array}{l}\text { No nexo entre o uso médico e o abuso potencial, as percepções } \\
\text { do público classificaram o CBD como semelhantes aos } \\
\text { analgésicos de venda livre, como Advil e Tylenol, e não } \\
\text { classificaram a maconha como uma substância de Classe I } \\
\text { Não houve diferenças nos tipos de medicamentos que eles } \\
\text { estavam substituindo por CBD e THC. }\end{array}$ \\
\hline Masataka, 2019. & $\begin{array}{l}\text { Efeitos ansiolíticos do tratamento } \\
\text { repetido com canabidiol em adolescentes } \\
\text { com transtornos de ansiedade social }\end{array}$ & $\begin{array}{l}\text { Os resultados indicam que o CBD pode ser uma opção útil para } \\
\text { tratar a ansiedade social }\end{array}$ \\
\hline Carvalho et al.,2020. & $\begin{array}{l}\text { A segurança, tolerabilidade e riscos } \\
\text { associados ao uso de medicamentos } \\
\text { antidepressivos de nova geração. }\end{array}$ & $\begin{array}{l}\text { Os resultados desta revisão devem alertar o médico para revisar } \\
\text { cuidadosamente a adequação da prescrição de antidepressivos } \\
\text { em uma base individual e considerar tratamentos alternativos, } \\
\text { se disponíveis. }\end{array}$ \\
\hline Turna et al., 2019. & $\begin{array}{l}\text { Comportamentos de uso de cannabis e } \\
\text { prevalência de ansiedade e sintomas } \\
\text { depressivos em uma coorte de usuários } \\
\text { canadenses de canabis medicinal }\end{array}$ & $\begin{array}{l}\text { Esses resultados destacam a necessidade de avaliar } \\
\text { sistematicamente o uso de CMP para doenças mentais. }\end{array}$ \\
\hline Berger et al., 2020. & $\begin{array}{l}\text { Tratamento do transtorno de ansiedade } \\
\text { social e sintomas psicóticos atenuados } \\
\text { com canabidiol }\end{array}$ & $\begin{array}{l}\text { Os achados deste estudo de caso acrescentam às evidências } \\
\text { existentes em apoio à segurança do CBD e sugerem que ele } \\
\text { pode ser útil para jovens com ansiedade refratária ao tratamento } \\
\text { e para sintomas psicóticos atenuados. }\end{array}$ \\
\hline Spechler et al., 2020. & $\begin{array}{l}\text { Ativações de recompensa atenuadas } \\
\text { associadas ao uso de canabis em } \\
\text { indivíduos ansiosos / deprimidos }\end{array}$ & $\begin{array}{l}\text { Esses dados apoiam a hipótese de que o uso de canabis em } \\
\text { indivíduos com transtornos de humor / ansiedade está associado } \\
\text { a um processamento cerebral atenuado de magnitude de } \\
\text { recompensa, o que pode contribuir para sintomas afetivos } \\
\text { persistentes. }\end{array}$ \\
\hline Bahji et al., 2020. & $\begin{array}{l}\text { Canabidiol: uma nova alternativa } \\
\text { potencial para o tratamento de ansiedade, } \\
\text { depressão e transtornos psicóticos. }\end{array}$ & $\begin{array}{l}\text { Esses resultados promissores apoiam o desenvolvimento de } \\
\text { estudos em larga escala para avaliar ainda mais o TCC como } \\
\text { uma nova droga em potencial para o tratamento desses } \\
\text { transtornos psiquiátricos }\end{array}$ \\
\hline $\begin{array}{l}\text { Chadwick et al., } \\
2020 .\end{array}$ & $\begin{array}{llrr}\text { Canabinoides } & \text { e } & \text { o } & \text { sistema } \\
\text { endocanabinóide na ansiedade, depressão } \\
\text { e desregulação da emoção em humanos. }\end{array}$ & $\begin{array}{l}\text { Este estudo cobre a investigação do canabidiol como } \\
\text { biomarcador potencial nessas condições. Isso é importante com } \\
\text { o aumento do uso medicinal de canabinoides e a crescente } \\
\text { tolerância social em relação ao uso recreativo de canabis. }\end{array}$ \\
\hline Black et al., 2019. & $\begin{array}{l}\text { Canabinoides para o tratamento de } \\
\text { transtornos mentais e sintomas de } \\
\text { transtornos mentais: uma revisão } \\
\text { sistemática e meta-análise }\end{array}$ & $\begin{array}{l}\text { Os resultados primários foram remissão e mudanças nos } \\
\text { sintomas desses transtornos mentais. }\end{array}$ \\
\hline Mandoli et al., 2018. & $\begin{array}{l}\text { Estudos clínicos realizados em pacientes } \\
\text { com diagnóstico de transtorno de } \\
\text { ansiedade social generalizada (TAS). }\end{array}$ & $\begin{array}{l}\text { Os autores mostraram que uma única dose de CBD não apenas } \\
\text { diminuiu significativamente os sintomas de ansiedade } \\
\text { subjetiva, mas também reduziram o comprometimento } \\
\text { cognitivo, o desconforto no desempenho da fala e o alerta na } \\
\text { fala antecipada durante um teste de simulação de fala em } \\
\text { Público. }\end{array}$ \\
\hline $\begin{array}{l}\text { Larsen e Shahinas., } \\
2020 .\end{array}$ & Estudos clínicos & $\begin{array}{l}\text { Administração oral aguda de } 600 \mathrm{mg} \text { (indivíduos com TAS) e } \\
300 \mathrm{mg} \text { (indivíduos saudáveis) de CBD reduziu a ansiedade } \\
\text { avaliada pela escala visual analógica de humor em comparação } \\
\text { com o placebo. }\end{array}$ \\
\hline
\end{tabular}




\begin{tabular}{|l|l|l|}
\hline Zuardi et al., 2017. & $\begin{array}{l}\text { Efeito ansiolítico do canabidiol (CBD) } \\
\text { em humanos. }\end{array}$ & $\begin{array}{l}\text { Os resultados confirmaram que a administração aguda de CBD } \\
\text { induziu efeitos ansiolíticos com uma curva em forma de U } \\
\text { invertido dependente da dose em indivíduos saudáveis, uma vez } \\
\text { que as medidas subjetivas de ansiedade foram reduzidas com } \\
\text { CBD 300 mg. }\end{array}$ \\
\hline Lindsey et al., 2021. & $\begin{array}{l}\text { Estudos in vitro e in vivo de interações } \\
\text { farmacocinéticas relevantes para o } \\
\text { tratamento de transtornos de ansiedade } \\
\text { em pessoas jovens. }\end{array}$ & $\begin{array}{l}\text { Em pacientes que tomam escitalopram, a adição de CBD } \\
\text { aumentou significativamente as concentrações plasmáticas de } \\
\text { escitalopram, ainda é incerto se isso também aumenta os } \\
\text { eventos adversos mediados por inibidores seletivos da } \\
\text { recaptação da serotonina. }\end{array}$ \\
\hline
\end{tabular}

Fonte: Autores.

Os estudos sugerem que, o uso do canabidiol demonstra ser seguro e eficaz para o tratamento da ansiedade, entretanto, boa parte dos estudos foram realizados em animais e a falta de estudos clínicos em humanos, em especifico com problemas de ansiedade tem dificultado a indicação por médicos, o uso agudo e crônico com doses variadas de CBD mostrou-se ser seguro (MacCallum \& Russo, 2018)

Um estudo com onze voluntários saudáveis tratados com $300 \mathrm{mg}$ (sete pacientes) e 600mg (quatro pacientes) de CBD oral em um estudo duplo-cego controlado por placebo mostrou que os níveis hormonais desses pacientes não faram alterados com uso do CBD, a via de administração pela qual foi administrada, farmacocinética, farmacodinâmica e efeitos fisiológicos também foram analisadas e obteve-se resposta positiva (Diest et al., 2019)

Um desenho cruzado, duplo-cego e de medidas repetidas em 16 homens voluntários saudáveis revelaram que, ao contrário de A 9-THC, o CBD não induziu sintomas psicóticos, sedação mental, deficiência intelectual ou sedação física em comparação com o placebo, a eficácia ansiolítica do CBD foi avaliada em pacientes com diagnóstico de transtornos de ansiedade (Gutiérres et al., 2020)

O óleo de CBD utilizado para o tratamento de doenças psiquiátricas apresenta baixo teor de THC e maior concentração de CBD, sendo o THC o responsável pelos efeitos eufóricos, em baixas quantidades não foi observado mudança pelos pesquisadores em baixas quantidades o THC potencializa o CBD, em um estudo realizado com uma paciente com diagnostico de ansiedade por trauma na infância demonstrou que o uso de $25 \mathrm{mg}$ do óleo diário por cinco meses reduziu drasticamente os sintomas de medo e esquivo ( Shannon et al., 2016)

Nestes estudos os autores descrevem os tipos de reações adversas e interações medicamentosas causadas pelo uso do canabidiol, entre as reações adversas foi citado: cansaço, alteração de apetite e ganho de peso. As interações medicamentosas identificadas mostrou que o canabidiol é metabolizado por meio da enzima CYP3A4 quando administrado por via oral, sendo assim, medicamentos como: cetoconazol, itraconazol, ritonavir e claritromicina inibem a atividade dessa enzima e pode levar a degradação do CBD, levando a concentrações séricas elevadas, ao contrario, fármacos como: fenobarbital, rifampicina, carbamazepina e fenitoina podem aumentar a atividade da enzima, aumentando sua excreção e diminuindo a concentração sérica (Iffland \& Grotenhermen, 2017)

Em pacientes que tomam citalopram ou escitalopram, a adição de CBD demonstrou um aumento significativamente as concentrações plasmáticas de citalopram, embora seja incerto se isso também aumenta os eventos adversos mediados por inibidores seletivos da recaptação da serotonina (Lyndsey et al., 2021)

O canabidiol demonstrou efeitos positivos, segurança e tolerabilidade em relação à resposta fisiológica e imunológica em camundongo e humanos, os parâmetros bioquímicos analisados não demonstrou alterações. Os estudos relatam que os efeitos farmacológicos podem variar conforme a posologia, entretanto, estudos indicam que a administração de dosagem baixa já causam efeito ansiolíticos por agirem em receptores canabinoides (CB 1 e CB 2 ), receptor 5-HT 1A (MacCallum \& Russo, 2018)

Os autores avaliaram os eventos adversos, tolerabilidade e segurança de inibidores seletivos da recaptação da serotonina, inibidores da recaptação da serotonina e noradrenalina, bupropiona, mirtazapina, trazodona, agomelatina, vilazodona, e 
vortioxetina. $\mathrm{O}$ estudo avaliou vários efeitos colaterais transitórios e podem desaparecer após algumas semanas após o início do tratamento, mas eventos adversos potencialmente graves podem persistir ou ocorrer posteriormente. Eles abrangem sintomas gastrointestinais como: náuseas, diarreia, sangramento gástrico, dispepsia, hepatotoxicidade, ganho de peso e anormalidades metabólicas, distúrbios cardiovasculares como: frequência cardíaca, hipertensão, hipotensão ortostática, e sintomas geniturinários (retenção urinária, incontinência) e disfunção sexual (Carvalho et al.,2020)

A administração de CBD pode exibir efeitos ansiolíticos agudos em pacientes com transtorno de ansiedade social generalizada através da modificação do fluxo sanguíneo cerebral em locais específicos do cérebro e agonista do receptor de serotonina (Zuardi et al.,2017; Mandoli et al., 2018)

O CBD demonstrou ser um composto seguro para a administração em seres humanos numa ampla faixa de dosagem e por vias diferentes. O uso em paciente por inalação também foi testado nos estudos, os pacientes começam com uma inalação e aguardam 15min. Então, eles podem aumentar em uma inalação a cada 15-30 min até que o controle dos sintomas desejado seja alcançado. Ha poucos estudos que demonstram essa via (MacCallum \& Russo, 2018)

Um estudo de caso forneceu dados que apoiam o uso de óleo de canabidiol como sendo seguro para reduzir a ansiedade e melhorar a qualidade de sono e transtorno pós-traumático de estresse. Em alta concentração, o CBD ativa diretamente o 5HT1A receptor de serotonina, conferindo assim um efeito antidepressivo. Estudos apoiam a eficácia do CBD como um ansiolítico, ant-ipsicótico e antidepressivo (Gutiérres et al., 2020)

A revisão de literatura sugere alguns benefícios do uso de CBD por causa de seu efeito ansiolítico e indutores do sono. Nove estudos com animais apoiam o uso deste tratamento e relataram que o CBD pode bloquear os sintomas de ansiedade induzida por movimento rápido dos olhos e alteração do sono por seu efeito ansiolítico no cérebro (Shannon et al., 2016)

Um estudo realizado no canada através de uma pesquisa por questionário online demonstrou que pacientes que fazem uso de antidepressivos e ansiolíticos disponíveis hoje trocaram os medicamentos atuais sem autorização medica por um tratamento com CBD, 2.032 respostas foram concluídas. Esses pacientes relatam buscar alternativa na medicina para substituir medicamentos atuais. Objetivo do estudo foi analisar a prevalência de canabis para fins medicinais. O estudo mostrou que a maioria (92\%) relatou que a canabis melhorou seus sintomas, apesar de continuar com gravidade de nível moderado (Turna et al., 2020)

Um estudo realizado com CBD para o tratamento da ansiedade e sintomas psicóticos demonstrou não estar claras no momento em relação ao tempo de tratamento e a dose utilizada. O estudo foi realizado com uma dose de $200 \mathrm{mg} /$ dia, que foi aumentada para $800 \mathrm{mg} /$ dia ao longo de 8 semanas. Melhora clinicamente significativa foi observada após 8 semanas de tratamento (Berger et al., 2020)

Estudos realizados em animais e humanos mostram que as propriedades de reforço da canabis são parcialmente devidas às interações com as vias de recompensa dopaminérgica no cérebro tipicamente alcançado durante a intoxicação aguda. Entretanto, os efeitos em longo prazo da canabis nos circuitos de recompensa têm poucas evidencias e precisam ser estudas (Spechler et al., 2020)

Os resultados de um estudo com 1.548 voluntários demonstraram que o canabidiol reduz os sintomas de ansiedade sem causar eventos adversos significativos. Maior eficácia foi observada entre os pacientes mais jovens e com tratamento mais longo, mesmo com sinais positivo o autor afirma que a necessidade de mais estudos clínicos (Bahji et al., 2020)

A partir dos estudos controlados foi possível identificar efeitos ansiolíticos com a administração aguda de CBD e efeitos terapêuticos para transtorno de ansiedade social, houve evidência para apoiar o efeito positivo de dose única no transtorno de ansiedade social (Lorsen \& Shahinas., 2020)

Evidências atuais sugerem que canabinóides, canabidiol ou preparações de canabis têm propriedades ansiolíticas. Entretanto, segundo os autores a depressão pode ser agravada pelo uso de canabis, mas, faltam ensaios clínicos randomizados 
para comprovar. O uso do canabidiol para PTSD (transtorno de estresse pós-traumático) mostrou várias linhas de evidência, essas apontam o sistema endocanabinóide como um jogador-chave em alguns dos transtornos revisados, em particular ansiedade e PTSD (Chadwick at al., 2020)

\section{Conclusão}

Segundo a OMS (organização mundial da Saúde), a depressão é um transtorno comum em todo o mundo, estima-se que mais de 300 milhões de pessoas sofram com ele. O potencial terapêutico da canabis para tratar uma variedade de condições neurológicas está se tornando cada vez mais reconhecidos mundialmente, vários países já legalizaram a canabis para uso medicinal e um número substancial de pacientes tem acesso ao medicamento. No Brasil a ANVISA (Agencia Nacional de Vigilância Sanitária) registrou o primeiro medicamento á base de canabidiol e THC, o Mevatyl em 2017 para tratamento da epilepsia, atualmente estudos vem sendo realizado para possível uso do canabidiol no tratamento da ansiedade.

Estudos mostram o canabidiol como uma potente opção terapêutica para o tratamento da ansiedade e depressão, esta revisão apresenta estudos com o óleo de CBD e seus benefícios, estudos clínicos mostram pouca reação adversa e boa tolerabilidade, entretanto, há necessidade de mais estudos com quantidade maior de voluntários, pois a maioria dos estudos disponíveis foi realizada em camundongo. Os estudos disponíveis apresentam uma quantidade baixa de testes em humanos e em uma população maior, há também uma necessidade de testes em população especifica como: idades diferentes, raça, sexo, portadores de doença crônica, com morbidade ou não, gravidas, efeitos toxicológicos, avaliação de função hepática e renal, taxas de hormônios precisam ser estudadas e mais estudos controlados com placebo.

Estudos em andamento mostram a eficácia do canabidiol e tetra-hidrocanabidiol no tratamento de transtorno estresse pós - traumático, transtorno de ansiedade social e transtorno de ansiedade generalizada. Vinte e quatro estudos foram identificados por esta revisão bibliográfica, dos quais vinte contribuíram para uma meta-análise com o objetivo de medir a eficácia e aceitabilidade de tratamentos com canabidinoide.

Ensaios grandes, duplo-cegos, randomizados e controlados por placebo são necessários para fornecer evidências de alta qualidade sobre a eficácia e aceitabilidade da canabis. A inibição do metabolismo hepático e da proteína responsável pelo transporte de fármacos por ex.: alfa-glicoproteína foi estudado apenas in vitro, por tanto, há uma necessidade de mais testes em humanos em relação a estes efeitos.

\section{Referências}

Alves, J. V.S., Waleria, P., Netto, P. R. R., Godman, B., Nascimento, R., C. R. M., \& Vital, W. C. (2021). Prevalence and factors associated with anxiety among university students of health sciences in Brazil: findings and implications. Jornal Brasileiro de Psiquiatria. 70(2) https://doi.org/10.1590/0047-2085000000322

Berger, M., Li, E., \& Amminger, G. P. (2020). Treatment of social anxiety disorder and attenuated psychotic symptoms with cannabidiol. BMJ Case Rep. 7,13(10): e235307 https://doi:10.1136/bcr-2020-235307

Bahji, A., Meyyappan, A. C., \& Hawken, E. R. (2020). Efficacy and acceptability of cannabinoids for anxiety disorders in adults. Journal of Psychiatric Research 129:257-264 https://doi.org/10.1016/j.jpsychires.2020.07.030

Black, N., Emily, S., Gabrielle, C., Lucy, T. T., Dino, Z., Wayne, D. H., \& Michael, F. (2019). Cannabinoids for the treatment of mental disorders and symptoms of mental disorders: Lancet Psychiatry. 6, P.995-1010, https://doi.org/10.1016/S2215-0366(19)30401-8

Blessing, E. M., Steenkamp, M. M., Manzanares, J., \& Marmar, C. R. (2015). Cannabidiol as a Potential Treatment for Anxiety Disorders. Neurotherapeutics. 12(4): $825-836$.

https://doi.org/10.1007/s13311-015-0387-1

Chadwick, V. L., Rohleder, C., Koethe, D., \& Leweke, F. M. (2020). Cannabinoids and the endocannabinoid system in anxiety, depression, and dysregulation of emotion in humans. Current Opinion in Psychiatry, 33(1), 20-42. https://doi:10.1097/YCO.0000000000000562 .

Carvalho, A. F., Sharma, M. S., Brunoni, A. R., Vieta, E., \& Fava, G. A. (2016). The Safety, Tolerability and Risks Associated with the Use of Newer Generation Antidepressant Drugs. Psychother Psychosom 85(5):270-88 https://doi:10.1159/000447034 
Dienst, W. K., Lewis, E. M., Buckner, J. D. (2019). Cannabis-Related Impairment and Social Anxiety: The Role of Use to Manage Negative and Positive Affect in Social Situations. Tailor \& Francis 55, Pages 271-280 https://doi:10.1080/10826084.2019.1664590

Gutiérrez-Garcia, M. S., Navarrete, F, Gasparyan, A, Austrich-Olivares, A., Sala, F. \& Manzanares, J. (2020). Cannabidiol: A Potential New Alternative for the Treatment of Anxiety, Depression, and Psychotic Disorders. Biomolecules 10(11):1575 https://doi:10.3390/biom10111575

Iffland, K. \& Grotenhermen, F. (2017). An Update on Safety and Side Effects of Cannabidiol: A Review of Clinical Data and Relevant Animal Studies. Cannabis Cannabinoid Res.1,2(1):139-154. https://doi:10.1089/can.2016.0034

Laczkouvis, C., Kothgassner, O.D., Felnhofer, A., \& Klier, C.M. (2021). Cannabidiol treatment in an adolescent with multiple substance abuse, social anxiety and depression. neuropsychiatrie 35, pages31-34 https://doi:10.1007/s40211-020-00334-0

Larsen, C., \& Shahinas, J. (2020). Dosage, Efficacy and Safety of Cannabidiol Administration in Adults: A Systematic Review of Human Trials. Journal of clinical medicine research, 12(3), 129-141. https://doi.org/10.14740/jocmr4090

Lyndsey, A. L., Doohan, P. T., Oldfield, Lachlan, M.S., Kevin, Richard, C., Arnold, Jonathon, C. P., Berger, Maximus, M.D, P., Amminger, G., Paul, M. D, P., McGregor, \& Iain, S.(2021). Citalopram and Cannabidiol, Journal of Clinical Psychopharmacology: 41 - p 525-533 https://doi:10.1097/JCP.0000000000001427

Masataka, N. (2019). Anxiolytic Effects of Repeated Cannabidiol Treatment in Teenagers With Social Anxiety Disorders. Journal: Frontiers in Psychology 10 P.2466 https://www.frontiersin.org/articles/10.3389/fpsyg.2019.02466/full

Mandolini, G., Lazzaretti, M., Pigoni, A., Oldani, L., Delvecchio, G., \& Brambilla, P. (2018). Pharmacological properties of cannabidiol in the treatment of psychiatric disorders: A critical overview. Epidemiology and Psychiatric Sciences, 27(4), 327-335. https://doi:10.1017/S2045796018000239

MacCallum, C. A., \& Russo, E. B. (2018). Practical considerations in medical cannabis administration and dosing. Eur J Intern Med. 49:12-19. https://doi:10.1016/j.ejim.2018.01.004

Mcfadden, Brandon, R, \& Malone, T. (2020). Homegrown perceptions about the medical use and potential abuse of CBD and THC. Addictive Behaviors 115, 106799 https://doi:10.1016/j.addbeh.2020.106799

Sakal, C., Lynskey, M., Schlag, A. K. \& Nutt, D. J. (2021). Developing a real-world evidence base for prescribed cannabis in the United Kingdom: preliminary findings from Project Twenty21.. Psychopharmacology. https://doi.org/10.1007/s00213-021-05855-2

Spechler, P. A., Jennifer, L. Stewart, Kuplicki, R, Jerzy, R., Sahib, J. S., Martin, R. P. Paulus, Jonathan J. A. B. F. K. K., Aupperle, R. L. ,Bodurka, J., Justin, S. , Feinstein, Sahib S. Khalsa, Martin, P. P., Jonathan, B. S., Jennifer, L. S., \& Teresa, A. V.(2020). Attenuated reward activations associated with cannabis use in anxious/depressed individuals. Journal of Psychiatric Research, Translational Psychiatry, 10:189 https://doi.org/10.1038/s41398-020-0807-9

Turna, J., William Simpson, B. P., \& Philippe Lucas, M. V. (2019). Ameringen Cannabis use behaviors and prevalence of anxiety and depressive symptoms in acohort of Canadian medicinal cannabis users. Journal of Psychiatric Research Volume 111, Pages: 134-139 https://doi.org/10.1016/j.jpsychires.2019.01.024

Wright, M., Ciano, P. D., \& Brands, B. (2020). Use of Cannabidiol for the Treatment of Anxiety: A Short Synthesis of Pre-Clinical and Clinical Evidence. Mary Ann Liebert, Inc., publishers. https://doi.org/10.1089/can.2019.0052

Zuardi, A. W., Rodrigues, N. P., Silva, A. L., Bernardo, S. A., Hallak, J. E. C., Guimarães, F. S., \& Crippa, J. A. S. (2017). Inverted U-Shaped Dose-Response Curve of the Anxiolytic Effect of Cannabidiol during Public Speaking in Real Life. Front Pharmacol. 11,8:259. https://doi:10.3389/fphar.2017.00259

Laboissière, P. (2017). No Dia Mundial da Saúde, OMS alerta sobre depressão. História ciências Saúde Manguinhos http://www.revistahcsm.coc.fiocruz.br/nodia-mundial-da-saude-oms-alerta-sobre-depressao/ 